Margrit Bischof, Friederike Lampert (Hg.)

Sinn und Sinne im Tanz 
Die Reihe wird herausgegeben von der Gesellschaft für Tanzforschung.

Margrit Bischof lebt in Bern und engagiert sich als Tanzdozentin und Tanzforschende für eine vielseitige Vernetzung von Forschung, Bildung und Kunst im Tanz. Sie konzipierte an der Universität Bern den Studiengang DAS/MAS TanzKultur.

Friederike Lampert (Prof. Dr. phil.) ist Professorin für Choreografie an der Zürcher Hochschule der Künste am Fachbereich Bachelor Contemporary Dance/ Master Dance. Ihr Schwerpunkt in der Lehre liegt in der Vermittlung von Tanzgeschichte durch choreografische Adaptionen. 
Margrit Bischof, Friederike Lampert (Hg.)

\section{Sinn und Sinne im Tanz}

Perspektiven aus Kunst und Wissenschaft

Jahrbuch TanzForschung 2020 
Dieses gtf-Jahrbuch entstand in Zusammenarbeit mit dem Institute for the Performing Arts and Film (IPF) der Zürcher Hochschule der Künste und erscheint als Band 20 der Reihe subTexte.

Die Reihe subTexte vereinigt Originaltexte zu jeweils einem Untersuchungsgegenstand aus den beiden Forschungsschwerpunkten»Performative Praxis«und»Film«. Sie bietet Raum für Texte, Bilder oder digitale Medien, die zu einer Forschungsfrage über, für oder mit Darstellender Kunst oder Film entstanden sind. Als Publikationsgefäß trägt die Reihe dazu bei, Forschungsprozesse über das ephemere Ereignis und die Einzeluntersuchung hinaus zu ermöglichen, Zwischenergebnisse festzuhalten und vergleichende Perspektiven zu öffnen. Vom Symposiumband bis zur Materialsammlung verbindet sie die vielseitigen, reflexiven, ergänzenden, kommentierenden, divergierenden oder dokumentierenden Formen und Ansätze der Auseinandersetzung mit den Darstellenden Künsten und dem Film.

\section{Bibliografische Information der Deutschen Nationalbibliothek}

Die Deutsche Nationalbibliothek verzeichnet diese Publikation in der Deutschen Nationalbibliografie; detaillierte bibliografische Daten sind im Internet über http://dnb.d-nb.de abrufbar.

\section{(c) $(1)(\Theta)$}

Dieses Werk ist lizenziert unter der Creative Commons Attribution-NonCommercial-NoDerivs 4.O Lizenz (BY-NC-ND). Diese Lizenz erlaubt die private Nutzung, gestattet aber keine Bearbeitung und keine kommerzielle Nutzung. Weitere Informationen finden Sie unter https://creativecommons.org/licenses/by-nc-nd/4.0/deed.de Um Genehmigungen für Adaptionen, Übersetzungen, Derivate oder Wiederverwendung zu kommerziellen Zwecken einzuholen, wenden Sie sich bitte an rights@transcript-publishing.com Die Bedingungen der Creative-Commons-Lizenz gelten nur für Originalmaterial. Die Wiederverwendung von Material aus anderen Quellen (gekennzeichnet mit Quellenangabe) wie z.B. Schaubilder, Abbildungen, Fotos und Textauszüge erfordert ggf. weitere Nutzungsgenehmigungen durch den jeweiligen Rechteinhaber.

\section{(C) 2020 transcript Verlag, Bielefeld}

Umschlaggestaltung: Maria Arndt, Bielefeld Umschlagabbildung: HAMONIM_PatriciaMai_@HannaNaske Lektorat: Margrit Bischof, Sabine Karoß Wissenschaftliche Beiträtinnen: Claudia Fleischle-Braun, Katja Schneider Satz: Mark-Sebastian Schneider, Bielefeld Druck: Majuskel Medienproduktion $\mathrm{GmbH}$, Wetzlar

Print-ISBN 978-3-8376-5340-3

PDF-ISBN 978-3-8394-5340-7

https://doi.org/10.14361/9783839453407

Gedruckt auf alterungsbeständigem Papier mit chlorfrei gebleichtem Zellstoff. Besuchen Sie uns im Internet: $h$ ttps://www.transcript-verlag.de Unsere aktuelle Vorschau finden Sie unter www.transcript-verlag.de/vorschau-download 